\title{
LORENTZ-INVARIANT HAMILTONIAN AND RIEMANN HYPOTHESIS
}

\author{
by \\ Susumu Okubo \\ Department of Physics and Astronomy \\ University of Rochester \\ Rochester, NY 14627, U.S.A.
}

\begin{abstract}
$\underline{\text { Abstract }}$
We have given some arguments that a two-dimensional Lorentz-invariant Hamiltonian may be relevant to the Riemann hypothesis concerning zero points of the Riemann zeta function. Some eigenfunction of the Hamiltonian corresponding to infinite-dimensional representation of the Lorentz group have many interesting properties. Especially, a relationship exists between the zero zeta function condition and the absence of trivial representations in the wave function.
\end{abstract}


The Riemann hypothesis ([1], [2], [3]) is one of the long-standing problems in the number theory. The Riemann's zeta function $\zeta(z)$ for a complex variable $z$ is defined for Re $z>1$ by

$$
\zeta(z)=\sum_{n=1}^{\infty} \frac{1}{n^{z}}
$$

and for other values of $z$ by its analytic continuation. It is well-known that $\zeta(z)$ is zero for negative even integer values of $z$, i.e. $z=-2,-4,-6, \ldots$, while all other non-trivial zeros of $\zeta(z)$ must lie in the strip $0<\operatorname{Re} z<1$. It has been conjectured that all non-trivial zeros of $\zeta(z)$ actually lie on the critial line $\operatorname{Re} z=\frac{1}{2}$. This Riemann hypothesis (hereafter referred to as $\mathrm{RH}$ ) is important in the number theory, since its validity can answer some questions concerning distributions of the prime numbers.

It has been suggested by many authors that the problem may be related to eigenvalue spectra of a self-adjoint operator $\mathrm{H}$ in some Hilbert space, although any such $\mathrm{H}$ has not been found so far. This view has been strengthened by the works of Odlyzko [4] and of others (see e.g. [5] and [6], and references quoted therein) that the statistical distributions of zero points of $\zeta(z)$ is consistent to a high degree with the law of the Gaussian unitary ensemble of random matrix theory [5], which is expected for spectra of complex Hamiltonians. Moreover, this fact is also found to be related to the phenomenon of the quantum chaos ([6] and [7]). A widely held opinion among many authors is that the validity of RH with its assoiciated Hamiltonian, if it exists, will shed light to the quantum chaos and vice versa.

The purpose of this note is to show the existence of one-parameter family of complex Hamiltonians which seems to be intimately connected with the problem. Moreover, these Hamiltonians are invariant under two-dimensional Lorentz transformation, a fact which will be of some intrinsic interest for its own right.

We start from the following integral representation [8] of $\zeta(z)$ :

$$
\zeta(z)=\frac{1}{\left(1-2^{1-z}\right) \Gamma(z)} \int_{0}^{\infty} d t \frac{t^{z-1}}{1+\exp t} \quad, \quad(\operatorname{Re} z>0)
$$

so that any non-trivial zero of $\zeta(z)$ must satisfy

$$
\int_{0}^{\infty} d t \frac{t^{z-1}}{1+\exp t}=0 \quad, \quad(1>\operatorname{Re} z>0)
$$


We call the condition Eq. (2) be zero zeta function condition (hereafter abbreviated as ZZFC). Also in view of the identity

$$
2^{1-z} \Gamma(z) \zeta(z) \cos \left(\frac{\pi}{2} z\right)=\pi^{z} \zeta(1-z),
$$

we may restrict ourselves to consideration only of the half sector $1>\operatorname{Re} z \geq \frac{1}{2}$ instead of $1>\operatorname{Re} z>0$ for ZZFC. Especially, if we can show that the assumption of $1>\operatorname{Re} z>\frac{1}{2}$ for $\zeta(z)=0$ will lead to a contradiction, this will prove $\mathrm{RH}$.

Suppose now that a Hamiltonian $\mathrm{H}$ is hermitian in a Hilbert space, so that we have

$$
<H \phi|\psi>=<\phi| H \psi>
$$

for wave functions $\phi$ and $\psi$. For a complex $z$ satisfying ZZFC, i.e. Eq. (2), we set

$$
z=\frac{1}{2}+i \lambda
$$

If $\mathrm{H}$ possessses a eigenfunction $\phi_{0}$ with the eigenvalue $\lambda$, i.e. if we have

$$
H \phi_{0}=\lambda \phi_{0} \quad,
$$

then Eq. (4) with $\psi=\phi=\phi_{0}$ will give $\lambda=\bar{\lambda}$ being real and hence $\operatorname{Re} z=\frac{1}{2}$, proving $\mathrm{RH}$. The natural question is whether such a $\mathrm{H}$ exists or not. Although we could not completely succeed, we have found some pairs $\left(H, \phi_{0}\right)$ satisfying the required condition Eq. (6), almost proving RH. The problem is that $\phi_{0}$ found so far appear to be not normalizable. However, a possibility exists that $\mathrm{H}$ may possess the correct eigenfunction $\phi_{0}$. Moreover, there exists an intriguing connection between ZZFC and the representation space of the Lorentz group under which $\mathrm{H}$ is invariant. These facts suggest that our Hamiltonian $\mathrm{H}$ may indeed still be relevant to the problem of $\mathrm{RH}$.

Let $\phi(x, y)$ and $\psi(x, y)$ be functions of two real variables $x$ and $y$. We introduce the inner product by

$$
<\phi \mid \psi>=\int_{-\infty}^{\infty} d x \int_{0}^{\infty} d y \bar{\phi}(x, y) \psi(x, y)
$$

Here and hereafter, $\bar{\phi}(x, y)$, for example, stands for the complex conjugate of $\phi(x, y)$. Note that the ranges of the integrations are $\infty>x>-\infty$ for $x$ but $\infty>y \geq 0$ for $y$. Consider a family of second-order differential operators given by

$$
H=\frac{\partial^{2}}{\partial x \partial y}+i \beta y \frac{\partial}{\partial y}+i(1-\beta) x \frac{\partial}{\partial x}+\frac{i}{2}
$$


for real parameter $\beta$. We note first that $\mathrm{H}$ is complex rather than real and second that it contains a purely imaginary constant term $i / 2$ whose presence is crucial for the hermiticity property of $\mathrm{H}$, as we will see below. By a simple calculation, it is easy to find

$$
\overline{(H \phi)} \psi-\bar{\phi}(H \psi)=\frac{\partial}{\partial x} J_{1}+\frac{\partial}{\partial y} J_{2}
$$

where we have set

$$
\begin{aligned}
J_{1} & =\frac{1}{2}\left(\frac{\partial \bar{\phi}}{\partial y} \psi-\bar{\phi} \frac{\partial \psi}{\partial y}\right)-i(1-\beta) x \bar{\phi} \psi \\
J_{2} & =\frac{1}{2}\left(\frac{\partial \bar{\phi}}{\partial x} \psi-\bar{\phi} \frac{\partial \psi}{\partial x}\right)-i \beta y \bar{\phi} \psi
\end{aligned}
$$

Note that the presence of the constant term $i / 2$ in the right side of Eq. (8) is pivotal in enabling to obtain Eqs. (9). Integrating both sides of Eq. (9), we will find the hermiticity condition Eq. (4), if we could discard all partially integrated terms involving $J_{1}$ and $J_{2}$. From the explicit expressions of $J_{1}$ and $J_{2}$ given above, this would be possible, if $\phi$ and $\psi$ or their derivatives with respect to $x$ vanish at $y=0$, and if $\phi$ and $\psi$ as well as their derivatives decrease sufficiently rapidly for $x \rightarrow \pm \infty$ and $y \rightarrow \infty$. Of course, we have to more carefully study the question of the domain and range of $\mathrm{H}$ in order to establish the self-adjointness of H. However, the naive criteria given above suffices for the present discussion. Especially, if $\phi$ satisfies Eq. (6), i.e.

$$
H \phi=\lambda \phi
$$

with the boundary condition

$$
\phi(x, 0)=0
$$

at $y=0$ and if $\phi(x, y)$ decreases rapidly at infinity, we will be able to establish $\mathrm{RH}$ in principle. We note that Eq. (10a) with $z=\frac{1}{2}+i \lambda$ implies the validity of

$$
\left\{\frac{\partial^{2}}{\partial x \partial y}+i \beta y \frac{\partial}{\partial y}+i(1-\beta) x \frac{\partial}{\partial x}\right\} \phi=-i z \phi
$$

We have yet to meaningfully utilize ZZFC in our formalism. Before going into its detail, we will first, however, note the following property of the Hamiltonian. $\mathrm{H}$ as well as the inner product $\langle\phi| \psi>$ are clearly invariant under the transformation

$$
x \rightarrow \frac{1}{k} x \quad, \quad \text { and } \quad y \rightarrow k y
$$


for any positive constant $k$. This invariance really reflects that of two-dimensional Lorentz transformation. To understand it better, consider new variables $u$ and $v$ given by

$$
x=u-v \quad, \quad y=u+v \quad .
$$

The Hamiltonian $\mathrm{H}$ is then invariant under the $\mathrm{SO}(1,1)$ Lorentz transformation

$$
\begin{aligned}
& u \rightarrow u^{\prime}=(\cosh \theta) u+(\sinh \theta) v, \\
& v \rightarrow v^{\prime}=(\sinh \theta) u+(\cosh \theta) v
\end{aligned}
$$

for real constant $\theta$, corresponding to the boost parameter $k=\exp \theta$. Because of the invariance, if $\phi$ satisfies $H \phi=\lambda \phi$, then so does $\phi\left(\frac{x}{k}, k y\right)$, and hence

$$
\widetilde{\phi}(x, y)=\int_{0}^{\infty} \frac{d k}{k} f(k) \phi\left(\frac{x}{k}, k y\right)
$$

for arbitrary function $f(k)$ satisfies also $H \widetilde{\phi}=\lambda \widetilde{\phi}$. Especially, any eigenfunction $\phi(x, y)$ of $\mathrm{H}$ may be regarded as a infinite-dimensional realization of the Lorentz group $\mathrm{SO}(1,1)$.

After these preparations, we will now discuss solutions of the differential equation (11). We have found the following two families of solutions. Let $g(\xi)$ be an arbitrary function of a variable $\xi$ which vanishes fast for $\xi \rightarrow \infty$. Then, we show first that

$$
\phi(x, y)=\int_{0}^{\infty} d t t^{z-1} \exp \left\{i x t^{1-\beta}\right\} g\left(t+y t^{\beta}\right) \quad, \quad(\operatorname{Re} z>0)
$$

with $\xi=t+y t^{\beta}$ is a solution of $H \phi=\lambda \phi$ with $z=\frac{1}{2}+i \lambda$. In this connection if we change $x \leftrightarrow y$ and $\beta \leftrightarrow 1-\beta$, it will also furnish a solution. This can be proved as follows. For simplicity, set

$$
G_{0}(x, y ; t)=\exp \left\{i x t^{1-\beta}\right\} g\left(t+y t^{\beta}\right)
$$

and note that $G_{0}$ satisfies a differential equation

$$
\left\{\frac{\partial^{2}}{\partial x \partial y}+i \beta y \frac{\partial}{\partial y}+i(1-\beta) x \frac{\partial}{\partial x}\right\} G_{0}=i t \frac{\partial}{\partial t} G_{0}
$$

as we can easily verify. Multiplying $t^{z-1}$ and integrating over $t$ from $t=\infty$ to $t=0$, then it reproduces Eq. (11) if $\operatorname{Re} z>0$. Especially, the special choice of

$$
g(\xi)=\frac{1}{1+\exp \xi}
$$


is of interest. Then, the function $f_{0}$ given by

$$
f_{0}(x, y)=\int_{0}^{\infty} d t \frac{t^{z-1}}{1+\exp \left[t+y t^{\beta}\right]} \exp \left(i x t^{1-\beta}\right)
$$

obeys

$$
H f_{0}=\lambda f_{0},
$$

although ZZFC implies only

$$
f_{0}(0,0)=0
$$

at the single point $x=y=0$, but not the desired boundary condition Eq. (10b) for arbitrary $x$. As we will see later, $f_{0}(x, y)$ is intimately related to the zeta function.

We can also find another class of solutions as follows. Let us consider now

$$
G_{1}(x, y ; u)=\frac{u^{\theta-1}}{(1-u)^{\theta}} e^{-i u x y} g\left(y u^{\beta}(1-u)^{1-\beta}\right)
$$

for a constant $\theta$ with $\xi=y u^{\beta}(1-u)^{1-\beta}$ for arbitrary function $g(\xi)$. We can verify that $G_{1}$ satisfies the differential equation

$$
\left\{\frac{\partial^{2}}{\partial x \partial y}+i \beta y \frac{\partial}{\partial y}+i(1-\beta) x \frac{\partial}{\partial x}\right\} G_{1}-i \frac{\partial}{\partial u}\left\{u(1-u) G_{1}\right\}=-i \theta G_{1} .
$$

Integrating Eq. (21) from $u=1$ to $u=0$, and assuming $1>\operatorname{Re} \theta>0$, it gives

$$
H f_{1}=\lambda_{1} f_{1}
$$

with

$$
\theta=\frac{1}{2}+i \lambda_{1}
$$

if we set

$$
f_{1}(x, y)=\int_{0}^{1} d u G_{1}(x, y, u)
$$

In order to obtain a solution which satisfies Eq. (10b), we let $x \rightarrow \frac{1}{k(t)} x$ and $y \rightarrow k(t) y$ for an arbitrary function $k(t)$ of a new variable $t$, and integrate Eq. (22c) on $t$ after multiplying $t^{z-1}(1+\exp t)^{-1}$. In this way, we generate a new family of solutions. In summary, the function

$$
\phi_{1}(x, y)=\int_{0}^{\infty} d t \frac{t^{z-1}}{1+\exp t} \int_{0}^{1} d u \frac{u^{\theta-1}}{(1-u)^{\theta}} e^{-i u x y} g(\xi)
$$


with

$$
\xi=k(t) y u^{\beta}(1-u)^{1-\beta}
$$

for arbitrary functions $k(t)$ of $t$ and $g(\xi)$ of $\xi$ is a solution of

$$
H \phi_{1}=\lambda_{1} \phi_{1} \quad, \quad\left(\theta=\frac{1}{2}+i \lambda_{1}\right)
$$

Moreover, if $g(0)=1$ (for example $g(\xi)=\exp (-\xi)$ ), then ZZFC will give the desired boundary condition

$$
\phi_{1}(x, 0)=0
$$

for $1 \geq \beta \geq 0$, since $y=0$ implies $\xi=0$. Therefore, with the choice of $\theta=z$ and hence $\lambda_{1}=\lambda$, the essential conditions Eqs. (10) will be obeyed for $\phi=\phi_{1}$. However, a difficulty is that it appears to lead to $\left\langle\phi_{1} \mid \phi_{1}\right\rangle=\infty$ in general, although a possibility may exist to avoid the dilemma for a suitable choice of $g(\xi)$. Instead of Eqs. (23), we may also use (by letting $u \rightarrow-u$ )

$$
\begin{aligned}
\phi_{1}(x, y) & =\int_{0}^{\infty} d t \frac{t^{z-1}}{1+\exp t} \int_{0}^{\infty} d u \frac{u^{\theta-1}}{(1+u)^{\theta}} e^{i u x y} g(\xi) \\
\xi & =k(t) y u^{\beta}(1+u)^{1-\beta}
\end{aligned}
$$

which satisfies Eq. (24) again. Eq. (25) can also be satisfied although the $u$-integration may logarthmically diverge at $u=\infty$ for $y=0$. Moreover $<\phi_{1} \mid \phi_{1}>$ could be even finite, at least if $\operatorname{Re} \theta>\frac{1}{2}$ for some $g(\xi)$. However it seems to be rather unlikely that the present $\phi_{1}$ can offer the correct wave function of the problem by the following reason: The solutions of Eqs. (23) belong to infinite-dimensional realizations of $\mathrm{SO}(1,1)$. Therefore, the given eigen-value $\lambda$ would then be infinitely degenerate because of the Lorentz covariance. We do not know how to resolve the dilemma. A simple way is to break the Lorentz invariance of $\mathrm{H}$ by adding a real non-covariant potential such as $\epsilon y$ for a constant $\epsilon$ or by letting $y \rightarrow y+\epsilon$ for Eq. (8). Nevertheless, there exists a indication that the present Hamiltonian $\mathrm{H}$ may not be completely irrelevant to $\mathrm{RH}$ as will be explained below.

The function $f_{0}(x, y)$ introduced by Eq. (18a) may also be related to RH by the following reason. We will first state without proof that there exist some constants $C_{0}, C_{1}, C_{2}$, 
and $C_{3}$ such that we have

$$
\begin{aligned}
& \left|f_{0}(x, y)\right| \leq C_{0} \\
& \left|f_{0}(x, y)\right| \leq C_{1} y^{-\frac{1}{\beta} \operatorname{Re} z} \\
& \left|f_{0}(x, y)\right| \leq C_{2}|x|^{-\frac{1}{1-\beta}} \operatorname{Re} z \\
& \left|f_{0}(x, y)\right| \leq C_{3}|x y|^{-\operatorname{Re} z}
\end{aligned}
$$

under the assumption of

$$
1>\beta>0
$$

Especially, if we have Re $z>\frac{1}{2}$, then $\left\langle f_{0}\right| f_{0}>$ is finite and the function $f_{0}(x, y)$ will furnish a infinite-dimensional unitary realization of the Lorentz group $\mathrm{SO}(1,1)$ with or without ZZFC. Moreover, if ZZFC is assumed, we will have first the orthogonality relation

$$
\int_{0}^{\infty} d x \int_{0}^{\infty} d y \bar{G}(x y) f_{0}(x, y)=\int_{-\infty}^{\infty} d x \int_{0}^{\infty} d y \bar{G}(x y) f_{0}(x, y)=0
$$

for arbitrary function $G(\xi)$ with $\xi=x y$, which will vanish sufficiently fast for $\xi \rightarrow \infty$. Second also under ZZFC, it satisfies a relation

$$
\int_{0}^{\infty} \frac{d k}{k} f_{0}\left(\frac{x}{k}, k y\right)=0
$$

We can show first Eq. (29) as follows. We rewrite the left side integral of Eq. (29) as

$$
J=\int_{0}^{\infty} \frac{d k}{k} f_{0}\left(\frac{x}{k}, k y\right)=\int_{0}^{\infty} d t t^{z-1} \int_{0}^{\infty} \frac{d k}{k} \frac{\exp \left(i \frac{x}{k} t^{1-\beta}\right)}{1+\exp \left[t+k y t^{\beta}\right]}
$$

and change the variable $k$ into $k \rightarrow k^{\prime}=k t^{\beta-1}$ to find

$$
J=\int_{0}^{\infty} d t t^{z-1} \int_{0}^{\infty} \frac{d k^{\prime}}{k^{\prime}} \frac{\exp \left(i x / k^{\prime}\right)}{1+\exp \left[\left(1+k^{\prime} y\right) t\right]}
$$

Interchanging the order of the integral and letting $t \rightarrow t^{\prime}=\left(1+k^{\prime} y\right) t$, this leads to

$$
J=\int_{0}^{\infty} \frac{d k^{\prime}}{k^{\prime}} \frac{\exp \left(i x / k^{\prime}\right)}{\left(1+k^{\prime} y\right)^{z}} \int_{0}^{\infty} d t^{\prime} \frac{\left(t^{\prime}\right)^{z-1}}{1+\exp t^{\prime}}
$$

which vanishes identically by ZZFC. 
Eq. (28) can then be shown by changing the variable $y$ into $k$ and then letting $x \rightarrow \xi=k x$ to calculate

$$
\begin{aligned}
\int_{0}^{\infty} d k \int_{0}^{\infty} d x \bar{G}(k x) f_{0}(x, k) & =\int_{0}^{\infty} d k \int_{0}^{\infty} \frac{d \xi}{k} \bar{G}(\xi) f_{0}\left(\frac{\xi}{k}, k\right) \\
& =\int_{0}^{\infty} d \xi \bar{G}(\xi) \int_{0}^{\infty} \frac{d k}{k} f_{0}\left(\frac{\xi}{k}, k\right)
\end{aligned}
$$

which is zero by Eq. (29).

The condition Eq. (29) can be interpreted to imply that the infinite-dimensional representation space of $\mathrm{SO}(1,1)$, spanned by $f_{0}(x, y)$ does not contain any singlet representation of the group. This is because the left side of Eq. (29) is precisely the Lorentz-invariant component contained in the representation space, since it is invariant under $x \rightarrow \frac{1}{\alpha} x$ and $y \rightarrow \alpha y$ for any positive constant $\alpha$. Then, the orthogonality relation Eq. (28) can be readily recognized to be the one between two mutually inequivalent representations of $\mathrm{SO}(1,1)$ since $G(x y)$ is clearly a Lorentz-scalar. Such a relationship between the condition $\zeta(z)=0$ and the absence of a trivial representation of $\mathrm{SO}(1,1)$ in $f_{0}(x, y)$ is quite intriguing and may indicate that $f_{0}(x, y)$ somehow plays a role in $\mathrm{RH}$.

We can find another type of orthogonality relation between two inequivalent eigenfunctions of $\mathrm{H}$. Let $\phi=\phi_{1}$ and $\psi=f_{0}$ in Eqs. (9) and integrate on $x$ and $y$. If we note $\phi_{1}(x, 0)=\frac{\partial}{\partial x} \phi_{1}(x, 0)=0$ at $y=0$, together with $H \phi_{1}=\lambda_{1} \phi_{1}$ and $H f_{0}=\lambda f_{0}$, it will lead to

$$
\int_{-\infty}^{\infty} d x \int_{0}^{\infty} d y \bar{\phi}_{1}(x, y) f_{0}(x, y)=0
$$

provided that we have $\bar{\lambda}_{1} \neq \lambda$.

In order to emphasize the dependence of $f_{0}(x, y)$ upon parameters $\beta$ and $z$, we now explicitly write it as

$$
F_{0}(x, y, z ; \beta)=\frac{1}{\Gamma(z)} \int_{0}^{\infty} d t \frac{t^{z-1}}{1+\exp \left(t+y t^{\beta}\right)} \exp \left(i x t^{1-\beta}\right)
$$

so that $f_{0}=\Gamma(z) F_{0}$ and it satisfies the differential equation

$$
\left\{\frac{\partial^{2}}{\partial x \partial y}+i \beta y \frac{\partial}{\partial y}+i(1-\beta) x \frac{\partial}{\partial x}\right\} F_{0}=-i z F_{0}
$$


as well as

$$
\frac{\partial}{\partial x} F_{0}(x, y, z ; \beta)=i F_{0}(x, y, z+1-\beta ; \beta)
$$

For special cases of $\beta=0$ and $\beta=1$, it reproduces the zeta function and its generalizations. For $\beta=1$, we change the integration variable $t$ into $t^{\prime}=(1+y) t$ and note Eq. (1) to obtain

$$
F_{0}(x, y, z ; 1)=\left(1-2^{1-z}\right) \zeta(z) \frac{e^{i x}}{(1+y)^{z}}
$$

For $\beta=0$, we calculate

$$
F_{0}(x, y, z ; 0)=e^{-y} \Phi\left(-e^{-y}, z, 1-i x\right)
$$

where $\Phi(\xi, z, \eta)$ is the generalized zeta function defined by

$$
\Phi(\xi, z, \eta)=\sum_{n=0}^{\infty}(\eta+n)^{-z} \xi^{n}
$$

which converges for $|\xi|<1, \eta \neq 0,-1,-2,-3, \ldots$ When we use the integral representation [8] of

$$
\Phi(\xi, z, \eta)=\frac{1}{\Gamma(z)} \int_{0}^{\infty} d t \frac{t^{z-1}}{1-\xi e^{-t}} e^{-\eta t}
$$

for $\operatorname{Re} \eta>0, \operatorname{Re} z>0,|\xi| \leq 1, \xi \neq 1$, and compare it with Eq. (31), we find Eq. (34). Since $F_{0}$ satisfies Eq. (32a), we see that $\Phi$ must be a solution of the differential equation

$$
\left\{\xi \frac{\partial^{2}}{\partial \xi \partial \eta}+\eta \frac{\partial}{\partial \eta}\right\} \Phi(\xi, z, \eta)=-z \Phi(\xi, z, \eta)
$$

which appears to have been overlooked in literature.

Eq. (37) enjoys symmetries larger than that for Eq. (11) for $\beta \neq 0$. It is first invariant under

$$
\text { (i) } \xi \rightarrow b \xi^{k} \quad, \quad \eta \rightarrow \frac{1}{k} \eta
$$

for any non-zero constants $k$ and $b$. Second, it also remains invariant under a transformation

(ii) $\Phi(\xi, z, \eta) \rightarrow \hat{\Phi}(\xi, z, \eta)=\xi^{\theta} \Phi(\xi, z, \eta+\theta)$ 
for another constant $\theta$. The case of $b=1$ in Eq. (38a) reflects the original Lorentz invariance Eq. (12). In this connection, identities

$$
\begin{aligned}
& \Phi(\xi, z, \eta)+\Phi(-\xi, z, \eta)=2^{1-z} \Phi\left(\xi^{2}, z, \frac{1}{2} \eta\right), \\
& \Phi(\xi, z, \eta)-\Phi(-\xi, z, \eta)=2^{1-z} \xi \Phi\left(\xi^{2}, z, \frac{1}{2}(\eta+1)\right),
\end{aligned}
$$

which can easily be verified from Eq. (35) are clearly consistent with the invariances under Eqs. (38) for special choices of $k=2, b= \pm 1$ and $\theta=\frac{1}{2}$.

In ending this note, we remark that a special case of $\beta=\frac{1}{2}$ may be of some interest. In that case, Eq. (8) becomes

$$
H=\frac{\partial^{2}}{\partial x \partial y}+\frac{i}{2}\left(x \frac{\partial}{\partial x}+y \frac{\partial}{\partial y}\right)+\frac{i}{2}
$$

which is now symmetric in $x$ and $y$. Applying a unitary transformation $H \rightarrow \widetilde{H}$ by

$$
\widetilde{H}=\exp \left(\frac{i}{2} x y\right) H \exp \left(-\frac{i}{2} x y\right)
$$

it is easy to find

$$
\widetilde{H}=\frac{\partial^{2}}{\partial x \partial y}+\frac{1}{4} x y
$$

Note first that the constant term $\frac{i}{2}$ in $\mathrm{H}$ has disappeared from Eq. (42). Second, $\widetilde{H}$ is real rather than complex, although this property is a special consequence only for $\beta=\frac{1}{2}$. Moreover, if we change the variables from $x$ and $y$ to $u$ and $v$ given by Eq. (13), i.e. $x=u-v$ and $y=u+v$, we can rewrite Eq. (42) now as

$$
\widetilde{H}=\frac{1}{4}\left(\frac{\partial^{2}}{\partial u^{2}}-\frac{\partial^{2}}{\partial v^{2}}+u^{2}-v^{2}\right)
$$

which represents two-dimensional anti-harmonic oscillator Hamiltonian in the sense that the signs of the quadratic potentials have the wrong signs in comparison to the harmonic oscillator case.

We have also found the following rather peculiar solution of $H \phi=\lambda \phi$ for the case of $\beta=\frac{1}{2}$. The function

$$
\phi(x, y)=\frac{\xi^{2 z}}{\xi^{2}+1} \exp \left(-\frac{i}{2} x \xi\right)
$$


with

$$
\xi=y+\left(1+y^{2}\right)^{\frac{1}{2}}
$$

can be shown to satisfy $H \phi=\lambda \phi$, although it may have nothing to do with the problem of $\mathrm{RH}$.

In conclusion, we have attempted in this note to present some arguments for possible relevance of our Hamiltonians to RH. Although they may not be the ultimate answer to the problem, there are at least some indications that they may be indirectly useful.

\section{Acknowledgement}

This paper is supported in part by the U.S. Department of Energy Grant no. DEFG02-91ER40685. 


\section{$\underline{\text { References }}$}

1. Titschmarsh, E.C.; 1951 "The Theory of the Riemann Zeta Function", (London, Oxford Unversity Press).

2. Edwards, H.M.; 1974 "Riemann Zeta Function", (New York, Academic Press).

3. Karatsuba, A.A.; 1995 "Complex Analysis in Number Theory", (Boca Raton, Florida, CRC Press).

4. Odlyzko, A.M.; 1987, Math. of Comp. 48, 273.

5. Mehta, M.L.; 1990 "Random Matrices and the Statistical Theory of Energy Level", revised and enlarged Second Edition, (New York, Academic Press).

6. Gutzwiller, M.C.; 1990 "Chaos in Classical and Quantum Physics", (New York, Springer Verlag).

7. Bolte, J.; 1993, Int. J. Mod. Phys. B7, 4451.

8. Erdélyi, A., Magnus, W., Oberhettinger, F., and Tricomi, F.G.; 1953 "Higher Transcendental Functions, Vol. I Bateman Manuscript Project", (New York, McGraw Hill). 